\title{
TIGHT EXTENSIONS OF NORMED SPACES
}

\author{
N. V. RAO
}

(Communicated by William J. Davis)

\begin{abstract}
In this note we show that bound extensions as defined by Kaufman (Acta Univ. (Szeged) 21 (1966), 163) and tight extensions as defined by Dress (Adv. in Math. 53 (1984), 322) are the same. Further we find that the property of being a bound extension is preserved under complexification.
\end{abstract}

\section{TIGHT $\Leftrightarrow$ BOUND}

The following definitions are taken from Kaufman [5, p. 163]. Unless explicitly stated otherwise, our considerations are valid in both categories-the category of complex normed spaces as well as the category of real normed spaces.

Definition 1.1. Let $X$ be a subspace of the normed space $Y$. Let $F$ be any subset of the closed unit ball $V$ of the dual space $Y^{*}$ of $Y . F$ is said to be an $X$-boundary if $\|x\|=\max _{f \in F}|f(x)|$ for every $x$ in $X$.

Definition 1.2. Let $X$ be a subspace of the normed space $Y . Y$ is said to be a bound extension of $X$ if every $X$-boundary is a $Y$-boundary.

Lemma 1.3 [5, Lemma 0, p. 163]. A normed space $Y$ is a bound extension of $X$ if and only if there exists no seminorm $N(y)$ on $Y$ except $\|y\|$ such that $N(y) \leq\|y\|$ for all $y$ in $Y$ and $N(x)=\|x\|$ for all $x$ in $X$.

Defintion 1.4 [2, p. 322]. Let $X$ be a metric subspace of the metric space $(Y, d) . \quad Y$ is said to be a tight extension of $X$ if given any pseudometric $\rho$ on $Y$ such that $\rho\left(y_{1}, y_{2}\right) \leq d\left(y_{1}, y_{2}\right)$ for every $y_{1}$ and $y_{2}$ in $Y$ and $\rho\left(x_{1}, x_{2}\right)=d\left(x_{1}, x_{2}\right)$ for every $x_{1}$ and $x_{2}$ in $X$, then $\rho\left(y_{1}, y_{2}\right)=d\left(y_{1}, y_{2}\right)$ for all $y_{1}, y_{2}$ in $Y$.

Remark 1.5. It is clear from the above definitions and the lemma that if $X$ is a subspace of the normed space $Y$ and $Y$ is a tight extension of $X$ as a metric space, then $Y$ is a bound extension of $X$ as a normed space.

Theorem 1.6. Suppose that $X$ is a subspace of a metric space $Y$ and

(a) $d\left(y, x_{1}\right)=\sup _{x \in X}\left(d\left(x_{1}, x\right)-d(y, x)\right)$ for all $y \in Y$ and all $x_{1} \in X$;

(b) $d\left(y_{1}, y_{2}\right)=\sup _{x \in X}\left|d\left(y_{1}, x\right)-d\left(y_{2}, x\right)\right|$ for all $y_{1}, y_{2} \in Y$.

Then $Y$ is a tight extension of $X$.

Received by the editors September 22, 1991.

1991 Mathematics Subject Classification. Primary 54E40, 46B99. 
Proof. Let $g$ be a metric on $Y$ extending $d$ on $X$ and subordinate to $d$ on $Y$.

By (a),

$$
\begin{aligned}
d\left(y, x_{1}\right) & =\sup _{x \in X}\left(d\left(x_{1}, x\right)-d(y, x)\right) \\
& \leq \sup _{x \in X}\left(g\left(x_{1}, x\right)-g(y, x)\right) \leq g\left(y, x_{1}\right) .
\end{aligned}
$$

Hence $g\left(y, x_{1}\right)=d\left(y, x_{1}\right)$ for all $y$ in $Y$ and all $x_{1}$ in $X$. Now by (b),

$$
\begin{aligned}
d\left(y_{1}, y_{2}\right) & =\sup _{x \in X}\left|d\left(y_{1}, x\right)-d\left(y_{2}, x\right)\right| \\
& =\left|g\left(y_{1}, x\right)-g\left(y_{2}, x\right)\right| \leq g\left(y_{1}, y_{2}\right) .
\end{aligned}
$$

So $d\left(y_{1}, y_{2}\right)=g\left(y_{1}, y_{2}\right)$ for all $y_{1}, y_{2}$ in $Y$. Thus $Y$ is a tight extension of $X$.

Theorem 1.7. If $Y$ is a bound extension of $X$ as a normed space, then $Y$ is a tight extension of $X$ as a metric space.

Proof. Let $V$ denote the closed unit ball of the dual $Y^{*}$ of $Y$, and let $F$ be a weak ${ }^{*}$-closed subset on which each of the functions $\hat{x} \quad(x \in X)$ restricted to $V$ attains its maximum modulus and no proper closed subset has the same property, i.e., $F$ is a minimal $X$-boundary. Existence of such minimal boundaries follows from Zorn's lemma and the weak * compactness of $V$. By hypothesis $F$ is also a $Y$-boundary. Let $\varepsilon(y)=\hat{y}$. Now $\varepsilon$ defines a linear isometry of $Y$ into $\mathscr{C}(F) . \mathscr{C}(F)$ denotes either the space of complex-valued continuous functions or real-valued continuous functions, depending on the category in which one is working.

By [5, Lemma 4, p. 165] it follows that

$$
\|f\|=\sup _{x \in X}(\|\hat{x}\|-\|\hat{x}+f\|)
$$

for all $f \in \mathscr{C}(F)$.

Further

$$
N(f-g)=\sup _{x \in X}|\|\hat{x}+f\|-\|\hat{x}+g\||
$$

for all $f, g$ in $\mathscr{C}(F)$ defines a seminorm extending $\|x\|$ on $X$ and subordinate to $\|f-g\|$ on $\mathscr{C}(F)$. Since $Y$ is a bound extension of $X$, it follows that

$$
N(\hat{y})=\|\hat{y}\| \text { for all } y \in Y .
$$

By (1.8), (1.9), and Theorem 1.6, $Y$ is a tight extension of $X$.

Corollary 1.10. $\mathscr{C}(F)$, as defined in the proof of Theorem 1.7, is a tight extension of $X$.

Proof. From (1.8) it immediately follows that $\mathscr{C}(F)$ is a bound extension of $X$ [5, Theorem 5, p. 165]. Hence from Theorem 1.7, $\mathscr{C}(F)$ is a tight extension of $X$. 


\section{Complexification}

Definition 2.1. Let $X$ be a real normed space and $U$ the closed unit ball of the dual space $X^{*}$. Let $X^{\mathrm{c}}$ be the set of all $x_{1}+i x_{2}$ where $x_{1}, x_{2}$ belong to $X$ and

$$
\left\|x_{1}+x_{2}\right\|=\sup _{\phi \in U}\left|\phi\left(x_{1}\right)+\phi\left(x_{2}\right)\right| .
$$

It is easy to see that $X^{\mathrm{c}}$ is a complex normed space and shall be referred to as the complexification of $X$.

Theorem 2.2. If $Y$ is an extension of $X$ in the category of real normed spaces, then $Y^{\mathrm{c}}$ is an extension of $X^{\mathrm{c}}$ in the category of complex normed spaces. If the former is a proper extension, then so is the latter.

The proof is obvious using the Hanach-Banach theorem.

Theorem 2.3. If $Y$ is a bound extension of $X$ in the category of real normed spaces, then $Y^{\mathrm{c}}$ is also a bound extension of $X^{\mathrm{c}}$ in the category of complex normed spaces and conversely.

Proof. Suppose that $Y$ is a bound extension of $X$ and $F$ is a minimal weak *closed boundary of $X$ in $U$, the closed unit ball of $X^{*}$. Let $G$ denote a minimal weak *-closed subset of the closed unit ball of $Y^{*}$ such that the natural epimorphism $\pi: Y^{*} \rightarrow X^{*}$ maps $G$ onto $F$. Such a $G$ would exist because of the Hahn-Banach theorem. Let $\mathscr{C}(G)=Z$, the space of complex-valued continuous functions on $G$ provided with the weak * topology. There exists a natural embedding of $Y^{\mathrm{c}}$ into $Z$, and further it is obvious that $G$ is a minimal closed boundary for $X^{\mathrm{c}}$. By [5, Theorem 5, p. 165], $Z$ is a bound extension of $X^{\mathrm{c}}$ and so $Y^{\mathrm{c}}$, being a subextension, is a bound extension of $X^{\mathrm{c}}$.

Now suppose that $Y$ is not a bound extension of $X$. Then there exists a closed subset $G$ of $V$, which is an $X$-boundary but not a $Y$-boundary. But then $G$ would be an $X^{\mathrm{c}}$-boundary but not a $Y^{\mathrm{c}}$-boundary, i.e., $Y^{\mathrm{c}}$ is not a bound extension of $X^{\mathrm{c}}$.

Theorem 2.4. Assume that $X$ is a real normed space. $X$ is injective in the category of real normed spaces if and only if $X^{\mathrm{c}}$ is injective in the category of complex normed spaces.

Proof. In any category, either real or complex, an object is injective if and only if it does not admit a proper bound extension. This is immediate from [5, Theorem 5, p. 165]. Hence if $X$ is not injective, it would admit a proper bound extension $Y$. But then $Y^{\mathrm{c}}$ would be a proper bound extension of $X^{\mathrm{c}}$ by Theorems 2.2 and 2.3. So $X^{\mathrm{c}}$ is not injective.

Suppose that $X$ is injective. By mimicking the classical proof of the HanachBanach theorem in the complex category we obtain that $X^{\mathrm{c}}$ is injective.

\section{Characterisation OF THE INJeCtive ENVElopes}

For any given compact Hausdorff space $T$, let $\mathscr{C}_{r}(T)$ denote the space of real-valued continuous functions on $T$ with the sup norm. Let $l(T)$ denote the space of all real-valued bounded functions on the set $T$ with the sup norm and let $\mathscr{B}(T)$ denote the smallest class of functions containing $\mathscr{C}_{r}(T)$ and contained in $l(T)$ such that it is closed under the operations $\sup f_{i}, \inf f_{i}$ where $\left\{f_{i}\right\}$ is any bounded family of functions in the class. 
Theorem 3.1. $\mathscr{B}(T)$ is the injective envelope of $\mathscr{C}_{r}(T)$ in two categories-the category of metric spaces and the category of real normed spaces. (For definitions refer to $[3,1]$.)

Proof. Since $\mathscr{B}(T)$ is closed under sup, inf operations over bounded families and contains the constant function 1 , by [6, Theorem 6, p. 45], it follows that it is injective. But then, by [5, Theorem 1, p. 163], there exists a subspace $Z$, which is injective and a bound extension of $\mathscr{C}_{r}(T)$. Again by the converse part of [6, Theorem 6], we have that $Z$ is closed under sup and inf. By the minimality of $\mathscr{B}(T), Z=\mathscr{B}(T)$. By Theorem $1.7, Z$ is a tight extension. Being tight and injective, $Z$ is the injective envelope in the category of metric spaces (see [2]). By construction, $Z$ is the injective envelope in the category of normed spaces.

Remark 3.2. No nonzero complex normed space can be injective in the real category.

Proof. Let $x$ be any nonzero element of the given space $X$. Let $\lambda=\frac{1+i \sqrt{3}}{2}$. Then the closed balls with radius $\|x\| / 2$, centers at $0, x$, and $\lambda x$ meet pairwise but do not have common intersection.

We may assume without loss of generality that $\|x\|=1$. By the Hahn-Banach theorem, there exists a complex linear functional $\phi$, such that $\phi(x)=1$, and $\|\phi\|=1$. Thus if the three balls above have a common point $\xi$, then

$$
\|\phi(\xi)\| \leq 1 / 2, \quad\|\phi(\xi)-1\| \leq 1 / 2, \quad\|\phi(\xi)-\lambda\| \leq 1 / 2 .
$$

From the first two inequalities, $\phi(\xi)=1 / 2$, and the last inequality gives $\|1 / 2-\lambda\| \leq 1 / 2$-a contradiction. Therefore $X$ cannot be hyperconvex [7], which is a necessary condition for being injective in the real category.

\section{EPILOGUE}

For more detailed bibliography we refer to [7] and the references cited there.

\section{REFERENCES}

1. Henry B. Cohen, Injective envelopes of Banach spaces, Bull. Amer. Math. Soc. 70 (1964), 723-726.

2. A. W. M. Dress, Trees, tight extension of metric spaces, and the cohomological dimension of certain groups: a note on combinatorial properties of metric spaces, Adv. in Math. 53 (1984), 321-402.

3. J. R. Isbell, Six theorems about injective metric spaces, Comment. Math. Helv. 39 (1964/ 1965), 65-74.

4. _ Injective envelopes of Banach spaces are rigidly attached, Bull. Amer. Math. Soc. 70 (1964), 727-729.

5. R. Kaufman, A type of extension of Banach spaces, Acta Univ. (Szeged) 21 (1966), 163-166.

6. Leopoldo Nachbin, Banach spaces with extension property, Trans. Amer. Math. Soc. 68 (1950), 28-46.

7. N. V. Rao, The metric injective hulls of normed spaces, Topology Appl. (to appear).

University of Toledo, Department of Mathematics, Toledo, OHio 43606

E-mail address: RNAGISE@UOFT02.UTOLEDO.EDU 